\title{
Essais
}

Revue interdisciplinaire d'Humanités

$17 \mid 2021$

Quels lieux pour les morts?

\section{Hiérarchie funéraire sous la dynastie des Tang}

Funerary Hierarchy in the Tang Dynasty

Jin Chen

\section{Q OpenEdition}

1 Journals

Édition électronique

URL : https://journals.openedition.org/essais/8530

DOI : 10.4000 /essais.8530

ISSN : 2276-0970

Éditeur

École doctorale Montaigne Humanités

Édition imprimée

Date de publication : 15 avril 2021

Pagination : 51-59

ISBN : 979-10-97024-09-3

ISSN : 2417-4211

Référence électronique

Jin Chen, « Hiérarchie funéraire sous la dynastie des Tang », Essais [En ligne], 17 | 2021, mis en ligne le 27 avril 2021, consulté le 18 janvier 2023. URL : http://journals.openedition.org/essais/8530 ; DOI :

https://doi.org/10.4000/essais.8530 


\section{Hiérarchie funéraire sous la dynastie des Tang}

\section{CHen Jin}

$\mathrm{La}$ vie et la mort sont les lois naturelles auxquelles l'homme ne peut échapper. Que ce soit l'empereur ou le premier ministre, les moines et les prêtres, les sages ou les ignorants, chacun, tôt ou tard, mourra. Après la mort d'un être humain, une série de rituels de pleurs, d'enterrement et de culte tenus par des parents et des amis imposent une dernière étiquette à la vie, les funérailles.

Depuis l'Antiquité, la Chine compte cinq types de rites fondamentaux : ceux de naissance, d'anniversaire, de couronne, ainsi que les rites nuptiaux et les rites funéraires. Parmi eux, la cérémonie d'anniversaire est une répétition de la commémoration de celle de naissance. Il n'y a donc en réalité que quatre sortes de grands rites durant la vie. Les anthropologues chinois pensent que le deuil provient de la peur du fantôme du défunt, et cela conduit au culte des ancêtres. Dans cet état d'esprit, le corps du défunt est traité d'une certaine manière et il existe un ensemble de rituels qui symbolisent l'affliction, dans l'espoir que le défunt atteindra un autre monde d'intérêt éternel. La façon dont les corps sont traités et éliminés varie selon les époques, les origines ethniques, les lieux et les classes sociales. Le système d'inhumation se compose principalement de l'inhumation céleste (qui consiste à exposer le cadavre aux oiseaux de proie), l'ensevelissement par immersion (mettant le corps dans les rivières et les lacs), la crémation, l'enterrement et ainsi de suite. Dans la société féodale chinoise, l'enterrement demeure la plus orthodoxe méthode du peuple Han et cela exprime leur croyance traditionnelle en « la paix dans le sol ».

La dynastie des Tang, à l'apogée de la société féodale chinoise, a été établie en 618 après J.-C. et a duré près de trois cents ans jusqu'à sa disparition en 907 après J.-C. C'est une période incontournable quand on parle de l'histoire médiévale de la Chine, parce que c'est une phase de transition historique cruciale où diverses religions, nationalités et cultures se sont heurtées et se sont finalement 
intégrées les unes dans les autres. Selon Chen Yinke ${ }^{1}$ dans son Esquisse d'un essai sur les origines des institutions Sui et Tang et Sur Han Yu, la première phase de la dynastie des Tang a vu la fin des désordres politiques, des perturbations sociales et des conflits étatiques depuis les dynasties du Nord et du Sud ${ }^{2}$, et puis à la phase suivante a été créée une nouvelle situation posant les fondements des Song et de la culture Han au sens où nous l'entendons aujourd'hui.

La capitale de la dynastie des Tang, Chang' ${ }^{3}{ }^{3}$, se situait dans la plaine de Guanzhong ${ }^{4}$. Les archéologues ont mis au jour des mausolée des empereurs des Tang et un grand nombre de tombes subordonnées aux alentours de la ville, dont la quantité totale s'élève jusqu'à plus de 3000 . Avec l'approfondissement et le raffinement de l'archéologie chinoise, les recherches sur les tombes des Tang sont majoritairement axées sur leurs mises en scène, leurs formes et leurs mobiliers funéraires. Ces études tendent davantage à l'analyse archéologique, en l'absence d'étude de la situation sociale, en particulier la relation entre le système social et la hiérarchie reflétée par le système de forme des tombes.

Dans cet article, nous discutons sur la hiérarchie funéraire et sociale sous la dynastie des Tang et essayons d'appréhender la transformation historique de la société féodale chinoise à l'époque.

\section{Hiérarchie funéraire}

La mort est un fait général, mais il en existe différentes expressions.

Selon Liji $i^{5}$, la mort de l'empereur se dit «Beng (崩)», la mort des princes "Hong (薨) », la mort des fonctionnaires érudits « $\mathrm{Zu}$ (卒) », la mort des lettrés non mandarins «Bulu (不禄) » et la mort du menu peuple «Si (死)». À

1 Chen Yinke, 陈寅恪, né le 3 juillet 1890 et mort le 7 octobre 1969, membre de l'Académie chinoise, est un historien chinois considéré comme l'un des plus créatifs et originaux du $\mathrm{XX}^{\mathrm{e}}$ siècle en Chine. Ses œuvres principales sont : Esquisse d'un essai sur les origines des institutions Sui et Tang (《隋唐制度渊源略论稿》), Esquisse de l'histoire politique des Tang ( 唐代政 治史述论稿》), et Biographie alternative de LIU Rushi (《柳如是别传》).

2 Les dynasties du Nord et du Sud, 南北朝 en chinois, de 420 après J.-C. à 589 après J.-C., ont succédé aux Seize Royaumes du nord et à la dynastie des Jin de l'Est du sud pour prendre fin en 589, avec la réunification par la dynastie des Sui qui leur a succédé. Mais la dynastie des Sui n'a connu que deux empereurs à cause de sa sévérité excessive. Les insurrections contre la tyrannie ne cessaient d'éclater jusqu'à la fin des Sui et l'avènement de la dynastie des Tang.

3 Chang'an, 长安, située à Xi'an (西安) d'aujourd'hui, est la capitale de plus de dix dynasties dans l'histoire de Chine, notamment les Tang. Le nom chinois de cette ville signifie littéralement « la paix permanente».

4 Guanzhong, 关中, ou la plaine de la Wei (渭河平原), est une région historique correspondant à la basse vallée de la Wei. Elle comprend la partie centrale de la province du Shaanxi (陕 西) ainsi que l'extrême ouest de l'actuel Henan. Le nom du Guanzhong date de la période des royaumes combattants et signifie littéralement " entre les passes".

5 Liji, ou Lijing, 《礼记》, le Classique des rites, désigne des ouvrages attribués aux sages de l'époque Zhou, compilés et commentés par les confucéens, traitant des rites encadrant l'organisation sociale, administrative et politique de cette dynastie. 
la dynastie des Tang, il est noté dans Kai Yuan $L i^{6}$ que quant au décès des fonctionnaires, "Hong " s'utilise pour ceux qui sont supérieurs au troisième degré, " $\mathrm{Zu}$ " pour ceux qui sont supérieurs au cinquième degré et " $\mathrm{Si}$ " pour tous ceux qui sont inférieurs au sixième degré, y compris le menu peuple.

La hiérarchie est un système social très important dans l'histoire de Chine. La dynastie des Tang au sommet de la société féodale chinoise pratiquait une hiérarchie plus complète et stricte qu'auparavant et qui a pénétré dans tous les aspects de la vie sociale, comme l'architecture, les vêtements, la nourriture, et les tombes ayant pour fonction de montrer le statut social du défunt de son vivant et de symboliser la vie de l'au-delà. Le grade de la propriété avant sa mort se reflète par la forme de sa tombe, la dimension du tombeau, la richesse de ses mobiliers funéraires.

En fonction des fouilles archéologiques, les tombes des Tang se divisent en trois catégories : les mausolées royaux, les sépulcres des fonctionnaires et les caveaux des civils. Les mausolées des empereurs et des membres royaux sont les plus magnifiques et ne correspondent pas forcément à une certaine loi. Les grades officiels des Tang se classent en neuf degrés. Quant aux sépulcres des fonctionnaires, il existe une réglementation stricte.

Superficie de la tombe sous la dynastie des Tang

\begin{tabular}{|c|c|c|c|c|c|c|c|}
\hline $\begin{array}{c}\text { Rang } \\
\text { Époque }\end{array}$ & $1^{\text {er }}$ degré & $2^{\mathrm{e}}$ degré & $3^{e}$ degré & $4^{\mathrm{e}}$ degré & $5^{\text {e degré }}$ & $\begin{array}{c}6^{\text {e degré }} \\
\text { et inférieur }\end{array}$ & civil \\
\hline Avant 741 & $\begin{array}{c}\text { Carré de } \\
90 \text { marches }\end{array}$ & $\begin{array}{c}\text { Carré de } \\
80 \text { marches }\end{array}$ & $\begin{array}{c}\text { Carré de } \\
70 \text { marches }\end{array}$ & $\begin{array}{c}\text { Carré de } \\
60 \text { marches }\end{array}$ & $\begin{array}{c}\text { Carré de } \\
50 \text { marches }\end{array}$ & $\begin{array}{c}\text { Carré de } \\
20 \text { marches }\end{array}$ & \\
\hline Après 741 & $\begin{array}{c}\text { Carré de } \\
70 \text { marches }\end{array}$ & \begin{tabular}{|c|} 
Carré de \\
60 marches
\end{tabular} & $\begin{array}{c}\text { Carré de } \\
50 \text { marches }\end{array}$ & $\begin{array}{c}\text { Carré de } \\
40 \text { marches }\end{array}$ & $\begin{array}{c}\text { Carré de } \\
30 \text { marches }\end{array}$ & $\begin{array}{c}\text { Carré de } \\
20 \text { marches }\end{array}$ & $\begin{array}{l}\text { Carré de } \\
\text { 7marches }\end{array}$ \\
\hline
\end{tabular}

Hauteur du tombeau sous la dynastie des Tang $^{7}$

\begin{tabular}{|c|c|c|c|c|c|c|c|}
\hline $\begin{array}{c}\text { Rang } \\
\text { Époque }\end{array}$ & $\mathbf{1}^{\text {er }}$ degré & $\mathbf{2}^{\text {e } \text { degré }}$ & $\mathbf{3}^{\text {e degré }}$ & $\mathbf{4}^{\text {e }}$ degré & $\mathbf{5}^{\text {e degré }}$ & $\begin{array}{c}\mathbf{6}^{\text {e degré }} \\
\text { et inférieur }\end{array}$ & civil \\
\hline Avant 741 & 1.8 zhang $^{1}$ & 1.6 zhang & 1.4 zhang & 1.2 zhang & 1 zhang & & \\
\hline Après 741 & 1.6 zhang & 1.4 zhang & 1.2 zhang & 1 zhang & 0.9 zhang & 0.8 zhang & 0.4 zhang \\
\hline
\end{tabular}

Tang Gao Zong ${ }^{8}$ a envoyé un rescrit à Li Yixuan, préfet de Yong Zhou, disant que les commerçants étaient devenus de plus en plus riches, qu'ils avaient commencé à effectuer des enterrements dispendieux, et qu'il lui faudrait arrêter ce phénomène de manière stricte. De cela, on comprend

6 Kai Yuan $L i$, 《开元礼》, ouvrage traitant des rites des officiels, promulgué en 732.

7 Zhang, 丈, unité de longueur chinoise qui revient à 3,3 mètres.

8 Li Zhi, 李治, né en 628 et mort en 683, dont le titre posthume est Tang Gao Zong (唐高宗), est le troisième empereur (de 649 à 683) de la dynastie des Tang. 
que l'envergure des tombes et le nombre de mobiliers funéraires n'étaient pas déterminés par la richesse personnelle. Tout le monde devait respecter strictement la hiérarchie du statut social.

La tombe peut être horizontale ou verticale. La tombe verticale est une fosse extraite du sol et la tombe horizontale une caverne creusée à une certaine profondeur puis excavée horizontalement. Dans la chambre mortuaire sont posés le cercueil et du mobilier funéraire. C'est la partie souterraine de la tombe. Sur le plan architectural, la chambre mortuaire est normalement construite en terre, en brique, en pierre ou même en bois. Sa forme et son échelle sont différentes en fonction de l'époque et des identités du propriétaire. La chambre en terre est fréquente sous la dynastie Sui et au début de la dynastie des Tang, et les tombes des hauts fonctionnaires ne font pas exception. Mais plus tard, les nobles préfèrent la chambre mortuaire en briques et celle en terre est prise plutôt par les civils et les officiels juniors.

Les quatre genres principaux de tombe des Tang se composent de la tombe en brique à double chambre en forme d'arc, la tombe en brique à une chambre en forme d'arc ou carrée, la tombe en terre à une chambre carrée, et la tombe en terre à une chambre rectangulaire. Dans la tombe en brique à double chambre reposent le prince, la princesse ou les exceptions autorisées par l'empereur. Les officiels du $1^{\text {er }}$ au $3^{\text {e }}$ degrés possèdent la tombe en brique à une chambre. Les propriétaires de la tombe en terre à une chambre carrée sont souvent les fonctionnaires de $4^{\mathrm{e}}$ et $5^{\mathrm{e}}$ degrés. Et ceux qui sont inférieurs au $6^{\mathrm{e}}$ degré, y compris les civils, n'ont que la tombe en terre à une chambre rectangulaire.

Qu'il s'agisse d'une chambre mortuaire en terre ou en brique, il y a toujours un passage qui y conduit. On voit généralement trois types de passage : type de pente longue, type de pente en marche, et type de puits vertical rectangulaire. À l'époque, les passages des tombes de l'aristocratie sont tous en pente, avec un long tunnel dont le sommet est ouvert comme un patio et les murs de deux côtés sont décorés de niches. Le nombre de patios et de niches correspond au titre officiel du propriétaire de la tombe. Selon les archéologues, la tombe avec plusieurs patios et niches murales symbolise le manoir de l'aristocrate féodal de son vivant.

En ce qui concerne le mobilier funéraire, un marché d'artefacts a été créé, ainsi qu'une institution dédiée à la fabrication d'artefacts exclusivement pour la cour impériale. Ces mobiliers comprennent principalement des figurines anthropomorphes et animales, des bêtes funéraires et des objets en poterie et en or ou argent. Aussi strictes sont les demandes sur la quantité, la dimension, la position de placement et même la disposition de la combinaison de ces objets funéraires utilisés. 
Le monde post mortem chez les Chinois des Tang a connu un système intégral influencé par le concept confucianiste de "Shen Zhong Zhui Yuan »?. Une des valeurs de base du confucianisme traditionnel est la piété filiale (孝). Elle s'exprime par deux situations : la piété filiale pour les parents vivants et celle pour des ancêtres y compris les parents morts. Les gens, de l'empereur à la population civile, étaient encouragés à servir les parents morts comme s'ils étaient encore vivants, parfois avec même plus d'attention. Jiang Chugong, issu d'une famille de mandarins, a gagné une réputation de piété filiale après avoir déménagé en plusieurs années les tombes de ses parents et grands-parents qui étaient dispersées dans différentes régions.

De plus, il est de notoriété publique que la dynastie des Tang est connue par sa puissance économique, sa stabilité sociale, son ouverture suprême vers l'extérieur et sa tolérance extrême. Il en a résulté une recrudescence des obsèques minutieuses et solennelles.

Les souverains, d'un côté, considéraient l'édification de leurs mausolées comme la priorité absolue de leur vivant ; de l'autre, ils attachaient de l'importance à la construction des sépulcres de leurs parents, tels que Xingning Ling ${ }^{10}$ et Shun Ling ${ }^{11}$. Les autres membres de la famille impériale et les fonctionnaires partageaient ce concept et prenaient les funérailles pour le plus important évènement de leur existence.

Mais il est à noter qu'à l'époque des Tang les mausolées des empereurs ont connu des développements historiques. Avant cette dynastie, les leaders de l'empire étaient parfois enterrés à côté de la tombe de leurs ministres influents. Leurs tombes sont toutes indépendantes et égales. La distinction est si peu évidente que les archéologues doivent faire référence aux découvertes de la fouille pour déterminer le statut du propriétaire. Par contre, les mausolées des empereurs des Tang sont devenus inviolables et sacrés et il n'y a plus de comparaison entre ces constructions gigantesques et toutes les autres tombes, y compris celles des princes héritiers.

À partir du fondateur LI Yuan ${ }^{12}$, la dynastie des Tang a connu au total 21 empereurs. Sauf les deux derniers empereurs, Li Hua enterré à Yinchi dans la province du Henan et Li Zhou inhumé à Heze dans la province du Shangdong, les 19 autres empereurs ont tous édifié leurs mausolées dans le

9 Shen Zhong Zhui Yuan, 慎终追远, est une citation dans les Entretiens de Confucius. Xue'er (“论语 ·学而》). La phrase complète est 《曾子曰: 慎终追远, 民德归厚矣 ", traduite par Séraphin Couvreur (1835-1919) : «Tseng tzeu dit : Si le prince rend les derniers devoirs à ses parents avec un vrai zèle et honore par des offrandes ses ancêtres même éloignés, la Vertu fleurira parmi le peuple».

10 Xingning Ling, 兴宁陵, est la tombe de Li Bing (李昞), père de Li Yuan.

11 Shun Ling, 顺陵, est la tombe de Madame Yang, mère de Wu Zhao.

12 Li Yuan, 李渊, né en 566 et mort en 635, dont le titre posthume est Tang Gao Zu (唐高祖), est le premier empereur de la dynastie des Tang. 
plateau au nord du fleuve Wei dans la province du Shaanxi. Ils sont devenus ce que l'on appelle aujourd'hui " les 18 mausolées des empereurs des Tang ", car dans Qian Ling ${ }^{13}$ reposent deux empereurs, Wu Zhao ${ }^{14}$, la seule impératrice régnante de toute l'histoire de Chine, et son époux Li Zhi.

Selon les statistiques archéologiques, Zhao $\operatorname{Ling}^{15}$ et Zhen Ling (贞 陵) mesurent respectivement $60 \mathrm{~km}^{2}$, Qian Ling et Tai Ling (泰陵) $40 \mathrm{~km}^{2}$, Xian Ling (献陵) $10 \mathrm{~km}^{2}$, et chacun de tous les autres 13 mausolées a une superficie de $20 \mathrm{~km}^{2}$.

Parmi les 18 mausolées, 14 sont dans les montagnes. On a creusé à mi-hauteur de la montagne choisie pour en faire la chambre mortuaire. Cette tradition date de l'époque de Li Shiming ${ }^{16}$, le deuxième empereur de la dynastie, et devient systématique et conventionnelle depuis Qian Ling. Li Shiming a démarré l'élévation de Zhao Ling en 636 dans la Montagne Jiu Zong ${ }^{17}$ et il a fallu 13 ans pour la finir. En outre, il y a plus de 200 tombes subordonnées distribuées en éventail devant Zhao Ling. L'immensité des travaux et la grandeur de l'édifice sont évidentes. Qian Ling est également réputé pour sa grande envergure et son élan majestueux ; sa construction a duré plus de 40 ans.

Nous constatons trois caractéristiques principales des mausolées des empereurs à l'époque. Premièrement, ils se sont majoritairement construits en profitant d'une montagne et la grande étendue spatiale se distingue plus que jamais des sépulcres des haut fonctionnaires. Deuxièmement, il y a toujours de magnifiques constructions au sol comme le parc funéraire entouré par des doubles murailles, les grandes voies sacrées et les sculptures de pierre spécifiques qui sont absolument interdites même aux tombeaux du premier ministre. Troisièmement, la richesse du mobilier funéraire, y compris des ouvrages d'orfèvrerie, des miroirs en laiton, des poteries et des porcelaines,

13 Qian Ling, 乾陵, se situe à l'actuel district de Qian (乾) à la ville de Xianyang dans la province du Shaanxi, à $85 \mathrm{~km}$ au nord-ouest de Xi'an. C'est le mieux sauvegardé des 18 mausolées impériaux des Tang.

14 Wu Zhao, 武昖, née en 624 et morte en 705 , est la seule impératrice régnante de toute l'histoire de Chine ; elle a régné de 690 à 705 ; son titre posthume est Zetian (则天). Le caractère Zhao (䁷) est inventé par elle-même pour signifier que le soleil (日) et la lune (月) brillent en même temps sur le ciel (空). La prononciation est identique à celle du mot «照 (éclairer) ". Elle cherchait une analogie avec la gouvernance conjointe de son couple.

15 Zhao Ling, 昭陵, se situe dans l'actuel district de Liquan (礼泉), dans la ville de Xianyang (咸阳), dans la province du Shaanxi. C'est un des sites historiques et culturels majeurs protégés au niveau national en Chine. Le musée de Zhao Ling a été fondé en 1979.

16 Li Shiming, 李世民, né en 600 et mort en 649, dont le titre posthume est Tang Tai Zong (唐 太宗), est le deuxième empereur (de 626 à 649) de la dynastie des Tang. Il est le second fils du premier empereur LI Yuan.

17 La montagne Jiu Zong, 九峻山, se situe à $22 \mathrm{~km}$ au nord-est du district de Liquan. Le sommet est à 1888 mètres d'altitude. 
ainsi que des fresques, doit correspondre aux lois et règlements. La quantité et la variété légales ne sont pas liées à la fortune du propriétaire de son vivant, mais uniquement à son statut social avant la mort.

\section{Transformation sociale}

La nette distinction des mausolées des empereurs s'explique par la centralisation suprême du pouvoir impérial.

Depuis la dynastie des Han, les institutions locales proposaient des talents au gouvernement central en fonction de leur érudition. Mais les connaissances à l'époque étaient transmises de père en fils au sein de la famille. Les descendants des anciennes familles honorables apprenaient à la fois les savoirs et la politique tous les jours dès le plus jeune âge. Pendant les dynasties du Nord, ces grandes familles à Guanzhong maîtrisaient également le pouvoir militaire, à l'instar du système tribal : il y avait un chef de chaque famille dirigeant sa propre tribu. Mais à leur tête existait un leader de tout le grand groupe, qui était bien sûr le chef de sa propre famille. Ces familles peuvent être considérées comme de petits groupes militaires armés. Les soidisant généraux dirigeaient leurs propres enfants.

À partir des dynasties des Jin, jusqu'au début de la dynastie des Tang, la Chine était donc une société dirigée par la noblesse. Le pouvoir de l'empereur ne pouvait être arbitraire et devait équilibrer et prendre en compte les intérêts des familles nobles qui l'entouraient. Le pouvoir impérial était décentralisé à ces grandes familles. Leur statut a été transmis de génération en génération et leur pouvoir ne cessait de se renforcer. Leur relation avec le peuple était très éloignée, comme entre des maîtres et des esclaves. Mais la distance qui les séparaient de l'empereur était aussi étroite que celle des frères.

Un empereur du début de la dynastie des Tang ne pouvait pas prendre une décision tout seul, parce qu'il représentait non seulement sa famille impériale de LI, mais aussi le groupe de noblesse et de pouvoir derrière lui. À son côté, il y avait 15 familles puissantes. CHen Yinke, l'un des plus célèbres historiens chinois, et Naito Hunan, grand spécialiste de l'histoire de Chine d'origine japonaise, ont tous analysé ce fait dans leurs travaux.

Au milieu de la dynastie des Tang, ces familles nobles étaient extrêmement corrompues, et alors des gens exclus de ce groupe de droits pendant longtemps ont commencé à les défier, y compris Wu Zhao. On a commencé à promouvoir les Jin Shi (进士) érudits venant de la classe ordinaire.

Quoique le système Ke Ju (科举) des examens officiels pour le recrutement des fonctionnaires ait été établi pendant la dynastie des Sui, celui-ci était tout différent du système des examens impériaux auquel nous pensons aujourd'hui. Il n'était pas très institutionnalisé, et la plupart du temps il était encore dépendant des recommandations par les locaux. Par ailleurs, les sujets d'examens étaient 
composés par le Ming Jing (明经) et le Ce Lun (策论). Pour celui-ci, il fallait rédiger une dissertation politique, et pour celui-là il fallait présenter des considérations sur les classiques confucianistes. Naturellement, c'étaient toujours les descendants des familles aristocratiques qui pouvaient réussir.

Au cours de la période Wu Zhao, a été portée une attention particulière aux lettrés ordinaires issus des familles pauvres. Ils étaient capables de passer l'examen de Jin Shi pour gagner un poste administratif, parce que le sujet d'examen de Jin Shi était simplement de rédiger un poème et un essai. C'est-à-dire que de grands poètes sans famille avaient finalement la chance de devenir des fonctionnaires. Ces gens ont été encouragés à lutter contre l'ancien groupe de pouvoir.

Au fil du temps, le plan de Wu Zhao a réussi. La plupart des fonctionnaires étaient à ce moment-là issus de la classe civile et l'ancien groupe de pouvoir a été détruit. Alors les fonctionnaires n'étaient plus aristocratiques et il n'y avait plus de protégés de l'empereur. Dans le passé, l'empereur avait été égal au chef de l'alliance familiale d'élite, il était maintenant tout seul au sommet du pouvoir en dirigeant des mandarins venus du menu peuple. Et il pouvait donc être plus dictatorial.

Cela a ouvert un nouveau chapitre de l'histoire de Chine où pendant la dynastie suivante des Song l'empereur dominait tout le monde et maîtrisait tout. Personne ne pouvait le provoquer. Même le premier ministre des Song n'était pas de la même classe que l'empereur. En revanche, le statut des civils s'est amélioré, car tout le monde avait la possibilité de se rendre au gouvernement par le biais d'un système d'examen plus équitable. Au cours de la dynastie des Song, il a été même dit que la noblesse ne durerait pas trois générations. Cela désigne le fait que même si leur grand-père et leur père étaient le premier ministre, les petits-enfants ne pouvaient pas être doués de pouvoir sans passer les examens impériaux.

Ainsi, la Chine a situé la fin de son Moyen Âge à la dynastie des Tang, et il y eut de grands changements historiques. Le déclin constant du statut de l'aristocratie et la montée du statut des civils nous empêchent d'entendre parler encore des grandes familles héréditaires. Plus important encore, grâce à l'intégration ethnique et culturelle à la dynastie des Tang, diverse ethnies, religions et cultures se sont heurtées et assimilées. Et cela a permis aux nouveaux confucianistes comme Zhu Xi (朱喜) apparus à la dynastie suivante de reconstruire un nouvel enseignement confucianiste.

\section{Conclusion}

Nous avons parlé des changements dans la hiérarchie funéraire sous la dynastie des Tang, mais il ne faut pas négliger les mutations politiques et sociales derrière ces changements de tombes. L'analyse du passé a pour objectif 
de mieux comprendre le présent et de réfléchir sur l'avenir. C'est après les Tang que nous voyons l'embryon de ce que l'on appelle « la Chine » et l'identité complète des Han reconnue aujourd'hui.

\author{
CHEN Jin \\ Université de Wuhan \\ crystal.chenjin@qq.com
}

\begin{abstract}
Résumé
Nous traitons ici de la liaison entre le développement de la hiérarchie funéraire et celui de la société chinoise de la dynastie des Tang. L'article est divisé en deux parties : la hiérarchie funéraire, et la transformation sociale. Ainsi, nous avons tenté d'aborder les changements de la hiérarchie funéraire à travers les changements sociaux et politiques de la dynastie des Tang. Nous en avons conclu que les litiges ainsi que les mutations politiques et sociales sont sousjacents à l'évolution concernant les rites funéraires et le développement des mausolées de cette période historique.
\end{abstract}

\title{
Mots-clés
}

Hiérarchie, funéraire, sociale, mausolée, dynastie des Tang.

\section{Abstract}

This article deals with the link between the development of the funeral hierarchy and the development of the Chinese society of the Tang Dynasty. Apart from the introduction and the conclusion, this article contains two parts, which respectively are the funeral hierarchy and the social transformation of the Tang Dynasty. Thus, we have attempted to explain the changes of the funeral hierarchy through the societal and political changes of the Tang Dynasty. We can conclude that political and societal changes underlie the evolution of funeral rites and the development of mausoleums of this historical period.

\section{Keywords}

Hierarchy, funeral, societal, mausoleum, Tang Dynasty. 